ARTICLE

\title{
Neutralization against Omicron SARS-CoV-2 from previous non-Omicron infection
}

Jing Zou ${ }^{1,9}$, Hongjie Xia (10 ${ }^{1,9}$, Xuping Xie (1) ${ }^{1,9}$, Chaitanya Kurhade ${ }^{1}$, Rafael R. G. Machado², Scott C. Weaver (iD ${ }^{2,3}$, Ping Ren (1D) ${ }^{4 凶} \&$ Pei-Yong Shi (iD $1,3,5,6,7,8 \times$

The spread of the Omicron SARS-CoV-2 variant underscores the importance of analyzing the cross-protection from previous non-Omicron infection. We have developed a high-throughput neutralization assay for Omicron SARS-CoV-2 by engineering the Omicron spike gene into an mNeonGreen USA-WA1/2020 SARS-CoV-2 (isolated in January 2020). Using this assay, we determine the neutralization titers (defined as the maximal serum dilution that inhibited $50 \%$ of infectious virus) of patient sera collected at 1 - or 6 -months after infection with non-Omicron SARS-CoV-2. From 1- to 6-month post-infection, the neutralization titers against USA-WA1/2020 decrease from 601 to 142 (a 4.2-fold reduction), while the neutralization titers against Omicron-spike SARS-CoV-2 remain low at 38 and 32, respectively. Thus, at 1- and 6-months after non-Omicron SARS-CoV-2 infection, the neutralization titers against Omicron are 15.8- and 4.4-fold lower than those against USA-WA1/2020, respectively. The low cross-neutralization against Omicron from previous non-Omicron infection supports vaccination of formerly infected individuals to mitigate the health impact of the ongoing Omicron surge.

\footnotetext{
${ }^{1}$ Department of Biochemistry and Molecular Biology, University of Texas Medical Branch, Galveston, TX, USA. ${ }^{2}$ Departments of Microbiology and Immunology, University of Texas Medical Branch, Galveston, TX, USA. ${ }^{3}$ Institute for Human Infection and Immunity, University of Texas Medical Branch, Galveston, TX, USA. ${ }^{4}$ Department of Pathology, University of Texas Medical Branch, Galveston, TX, USA. ${ }^{5}$ Sealy Institute for Drug Discovery, University of Texas Medical Branch, Galveston, TX, USA. ${ }^{6}$ Institute for Translational Sciences, University of Texas Medical Branch, Galveston, TX, USA. ${ }^{7}$ Sealy Institute for Vaccine Sciences, University of Texas Medical Branch, Galveston, TX, USA. ${ }^{8}$ Sealy Center for Structural Biology \& Molecular Biophysics, University of Texas Medical Branch, Galveston, TX, USA. ${ }^{9}$ These authors contributed equally: Jing Zou, Hongjie Xia, Xuping Xie. ${ }^{凶}$ email: piren@utmb.edu; peshi@UTMB.edu
} 
S evere acute respiratory syndrome coronavirus 2 (SARS$\mathrm{CoV}-2$ ) continues to evolve, leading to the emergence of variants of concern $(\mathrm{VoC})$, variants of interest, and variants of monitoring. These variants can increase viral transmission, immune evasion, and/or disease severity ${ }^{1-3}$. The recently emerged Omicron variant (B.1.1.529) was first identified in South Africa on November 2, 2021, and was designated as a new VoC on November 26, along with the four previous VoCs: Alpha, Beta, Gamma, and Delta ${ }^{4}$. Since its emergence, Omicron has rapidly spread to over 89 countries, with case doubling in as little as 1.5 to 3 days, leading to global surges of COVID-19 cases $^{5}$. Compared to prior variants, the Omicron spike glycoprotein has accumulated more spike mutations, with over 34 mutations, many of which are known to evade antibody neutralization (e.g., $\mathrm{K} 417 \mathrm{~N}$, N440K, S477N, E484A and Q493R) or to enhance spike/hACE2 receptor binding (e.g., Q498R, N501Y, and D614G) $)^{1,3,6,7}$. The high number of spike mutations is associated with decreased potency of antibody therapy and increased breakthrough Omicron infections in vaccinated and previously infected individuals ${ }^{5}$. Laboratory studies are needed to examine the susceptibility of Omicron SARS-CoV-2 to vaccine- and infection-elicited neutralization. This study aimed to examine the cross-neutralization of Omicron by antibodies derived from previous non-Omicron infection.

\section{Results and discussions}

To measure neutralization of the Omicron variant, we developed a high-throughput assay. Using a previously established mNeonGreen (mNG) reporter USA-WA1/2020 SARS-CoV-2 ${ }^{8}$, we swapped the original spike gene with an Omicron spike (BA.1 lineage; GISAID EPI_ISL_6640916), resulting in recombinant mNG Omicron-spike SARS-CoV-2 (Supplementary Fig. 1). The mNG gene was placed at the open-reading-frame-7 (ORF7) of the viral genome 9 . The engineered Omicron spike contained mutations A67V, H69-V70 deletion ( $469-70)$, T95I, G142D, V143Y145 deletion $(\Delta 143-145)$, N211 deletion $(\Delta 211)$, L212I, L214 insertion EPE (Ins214EPE), G339D, S371L, S373P, S375F, K417N, N440K, G446S, S477N, T478K, E484A, Q493R, G496S, Q498R, N501Y, Y505H, T547K, D614G, H655Y, N679K, P681H, N764K, D796Y, N856K, Q954H, N969K, and L981F (Supplementary Fig. 1). The mNG Omicron-spike virus was sequenced to ensure no undesired mutations. After infecting Vero E6 cells, parental mNG USA-WA1/2020 developed larger fluorescent foci than Omicron-spike SARS-CoV-2 (Supplementary Fig. 2); the smaller fluorescent foci might be caused by the less fusogenic ability of the Omicron spike glycoprotein; however, comparable infectious titers of $>10^{6}$ focus-forming units per milliliter (FFU/ $\mathrm{ml}$ ) were obtained for both viruses. The $\mathrm{mNG}$ viruses were used to develop a fluorescent focus reduction neutralization test (FFRNT) as depicted in Supplementary Fig. 3.

We examined the cross-neutralization of non-Omicron SARSCoV-2-infected patient sera against Omicron virus. Two panels of COVID-19 patient sera, one collected at 1-month post-infection $(n=64)$ and another collected at 6 -month post-infection $(N=36)$, were measured for their $50 \%$ fluorescent focus reduction neutralization titers $\left(\mathrm{FFRNT}_{50}\right.$, defined as the maximal dilution that neutralized $50 \%$ of foci) against both USA-WA1/ 2020 and Omicron-spike SARS-CoV-2. Supplementary Tables 1 and 2 summarize the patient information (e.g., age, gender, race, date of positive viral test, symptom, and hospitalization) for the 1and 6-month postinfection serum panels. All patients were infected before February 2021, prior to the emergence of the Omicron variant. The 1-month postinfection sera neutralized USA-WA1/2020 and Omicron-spike SARS-CoV-2 with geometric mean titers (GMTs) of 601 and 38, respectively (Fig. 1a and Supplementary Fig. 4a). Only one serum had a neutralization titer of $<20$ against USA-WA1/2020, whereas 23 of 64 sera had neutralization titers of $<20$ against Omicron-spike SARS-CoV-2 (Fig. 1a). Sera with high neutralization titers against USA-WA1/ 2020 were from symptomatic patients, most were hospitalized (Supplementary Table 1), confirming that neutralizing antibody levels are associated with COVID-19 disease severity ${ }^{10}$. Notably, many of the sera with the highest neutralization titers of $>3450$ against USA-WA1/2020 were from patients who had received convalescent plasma treatment (Supplementary Table 1).

The 6-month postinfection sera neutralized USA-WA1/2020 and Omicron-spike SARS-CoV-2 with GMTs of 142 and 32, respectively (Fig. 1b and Supplementary Fig. 4b). Consistent with the 1-month post-infection results, symptomatic hospitalized patients tended to have higher neutralization titers against USAWA1/2020 than asymptomatic individuals (Supplementary Table 2). Thus, from 1- to 6-months post-infection, the mean neutralization titers against USA-WA1/2020 waned from 601 to 142 (a 4.2-fold decrease), while the neutralization titers against Omicron-spike virus remained low and nearly unchanged at 38 and 32 , respectively. Consistent with our results, the waning neutralization overtime against non-Omicron SARS-CoV-2 was previously reported in naturally infected or vaccinated individuals ${ }^{1-13}$. Our data showed that 1- and 6-months after non-Omicron SARS-CoV-2 infections, the neutralization titers against Omicron were 15.8- and 4.4-fold lower than those against USA-WA1/2020, respectively. A similar range of neutralization reduction against the Omicron virus was reported for two-dose mRNA-vaccinated sera ${ }^{14-16}$. However, there is a correlation between the neutralization titers against USA-WA1/2020 and those against Omicron (i.e., sera with high neutralization titers against USA-WA1/2020 also exhibiting high neutralization titers against Omicron; Supplementary Figs. 4 and 5). Collectively, these results demonstrate low cross-neutralization against the Omicron variant from previous non-Omicron viral infection or two-dose mRNA vaccination. The low cross-neutralization against the Omicron variant strongly suggests that individuals previously infected with SARS-CoV-2 should be vaccinated to mitigate Omicron-mediated infection, disease, and/or potential death.

Among all tested sera, only 6 pairs of 1- and 6-month samples were collected from same individuals (Supplementary Tables 1 and 2). Their neutralization patterns (Supplementary Fig. 5) were similar to those observed with the means from complete 1- and 6-month serum panels.

Our study has several limitations. First, we have not defined the contributions of individual spike mutations to Omicron neutralization evasion. The constellation of Omicron spike mutations may result from selection for either viral transmission, immune escape, or both. The emergence of the Omicron variant in South Africa, where herd immunity is believed to be high, is consistent with evolutionary pressure for immune escape as suggested by our data and others ${ }^{17}$. Second, the genotypes of viruses that infected the patients whose sera were analyzed in this study were not defined. Third, we have not analyzed other immune modulators. $\mathrm{CD}^{+} \mathrm{T}$ cells and non-neutralizing antibodies that can mediate antibody-dependent cytotoxicity are known to protect patients from severe disease. The Omicron spike mutations may not dramatically affect $\mathrm{T}$ cell epitopes ${ }^{18}$.

The rapid evolution of SARS-CoV-2 underscores the importance of surveillance for new variants and their impact on viral transmission, disease severity, and immune evasion. Surveillance, laboratory investigation, and real-world vaccine effectiveness are essential to guide if and when an Omicron-specific vaccine or booster is needed. Currently, vaccination with booster shots ${ }^{19,20}$, together with masking and social distance, remain to be the most effective means to mitigate the health impact of Omicron surge. 
a

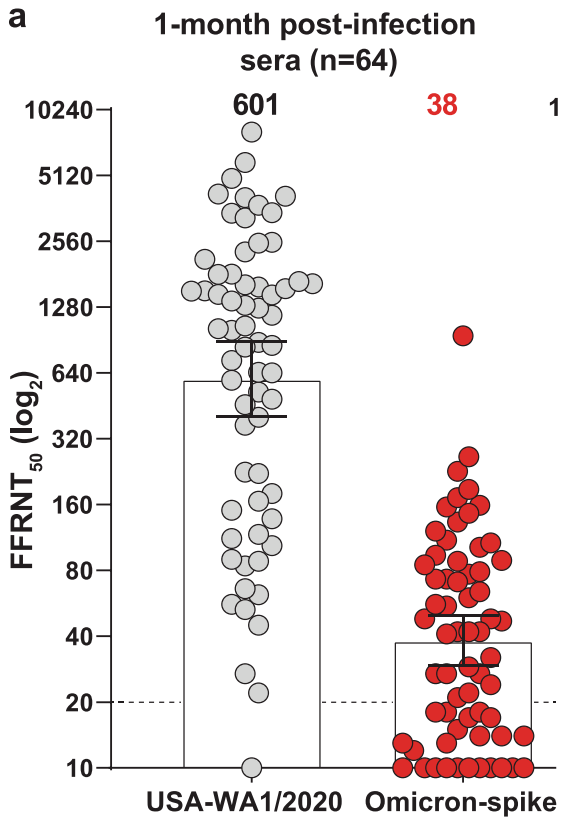

b

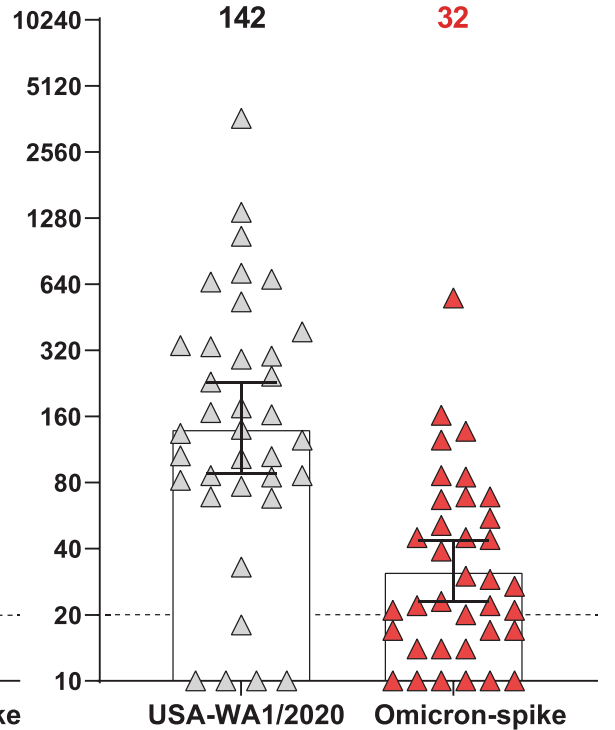

Fig. 1 Reduced neutralization of Omicron SARS-CoV-2 by previous non-Omicron viral infection. 50\% fluorescent focus reduction neutralization titers $\left(\right.$ FFRNT $_{50}$ ) were measured for two serum panels from patients previously infected with non-Omicron SARS-CoV-2. The first serum panel was collected at 1-month post-infection $(n=64)$ and the second panel collected at 6 -months post-infection $(n=36)$. For each serum, FFRNT 50 values were determined against mNG USA-WA1/2020 (in gray color) and Omicron-spike SARS-CoV-2 (in red color). a FFRNT ${ }_{50}$ S of 1-month postinfection sera (shown as circles). b FFRNT ${ }_{50}$ s of 6 -month postinfection sera (shown as triangles). Supplementary Tables 1 and 2 summarize the FFRNT 50 values and serum information for (a) and (b), respectively. Each symbol of dots (a) and triangles (b) represents one serum specimen. The FFRNT 50 value for each serum was determined in duplicate assays and is presented as the geometric mean. The bar heights and the numbers above each set of data indicate geometric mean titers. The whiskers indicate $95 \%$ confidence intervals. The dotted line indicates the first serum dilution (1:20) of the FFRNT assay. The FFRNT 50 values of sera that did not show any inhibition of viral infection are presented as 10 for plot purposes and statistical analysis. Statistical analysis was performed using the Wilcoxon matched-pairs signed-rank test. The statistical significance of the difference between the geometric mean titers against USA-WA1/2020 and Omicron-spike SARS-CoV-2 is $p<0.0001$ in both (a) and (b).

Finally, the high-throughput fluorescent neutralization assay reported in this study can expedite therapeutic antibody screening, neutralization testing, and modified vaccine development.

\section{Methods}

Cells. Vero E6 (ATCC ${ }^{\oplus}$ CRL-1586) were purchased from the American Type Culture Collection (ATCC, Bethesda, MD), and maintained in a high-glucose Dulbecco's modified Eagle's medium (DMEM) supplemented with $10 \%$ fetal bovine serum (FBS; HyClone Laboratories, South Logan, UT) and 1\% penicillin/ streptomycin at $37^{\circ} \mathrm{C}$ with $5 \% \mathrm{CO}_{2}$. All culture medium and antibiotics were purchased from ThermoFisher Scientific (Waltham, MA). The cell line was tested negative for mycoplasma.

Construction of recombinant viruses. The recombinant mNeoGreen (mNG) Omicron-spike SARS-CoV-2 was constructed on the genetic background of an infectious cDNA clone derived from clinical strain WA1 (2019-nCoV/USA_WA1/ 2020) containing an $m N G$ reporter gene ${ }^{9}$. The Omicron spike mutations, including A67V, $469-70$, T95I, G142D, $\Delta 143-145, \Delta 211$, L212I, Ins214EPE, G339D, S371L, S373P, S375F, K417N, N440K, G446S, S477N, T478K, E484A, Q493R, G496S, Q498R, N501Y, Y505H, T547K, D614G, H655Y, N679K, P681H, N764K, D796Y, N856K, Q954H, N969K, and L981F, were engineered using a PCR-based mutagenesis protocol as reported previously ${ }^{1}$. Supplementary Table 3 listed all the primers for the mutagenesis. The full-length genomic cDNAs were in vitro ligated and used as templates to transcribe full-length viral RNA using the mMESSAGE mMACHINE ${ }^{m}$ T7 Transcription Kit (ThermoFisher Scientific). Mutant viruses were recovered on day 3 after Vero E6 cells were electroporated with the in vitro RNA transcripts. The harvested virus stocks were quantified for their infectious titers (fluorescent focus units) by titrating the viruses on Vero E6 cells in a 96-well plate after $16 \mathrm{~h}$ of infection. The genome sequences of the virus stocks were confirmed to have no undesired mutations by Sanger sequencing. The detailed protocol of genome sequencing was recently reported ${ }^{21}$. All virus work was performed in a biosafety level 3 (BSL-3) laboratory with redundant fans in the biosafety cabinets at The University of Texas Medical Branch at Galveston. All personnel wore powered air purifying respirators (Breathe Easy, $3 \mathrm{M}$ ) with Tyvek suits, aprons, booties, and double gloves.
Serum specimens. The research protocol regarding the use of human serum specimens was reviewed and approved by the University of Texas Medical Branch (UTMB) Institutional Review Board (IRB\#: 20-0070). The de-identified convalescent sera from COVID-19 patients (confirmed by the molecular tests with FDA's Emergency Use Authorization) were heat-inactivated at $56^{\circ} \mathrm{C}$ for $30 \mathrm{~min}$ before testing. No informed consent was required since these deidentified sera were leftover specimens before being discarded. No diagnoses or treatment was involved either.

Fluorescent focus reduction neutralization test. Neutralization titers of human sera were measured by a fluorescent focus reduction neutralization test (FFRNT) using the mNG reporter SARS-CoV-2. Briefly, Vero E6 cells $\left(2.5 \times 10^{4}\right)$ were seeded in each well of black $\mu$ CLEAR flat-bottom 96-well plate (Greiner Bio-one ${ }^{\mathrm{rm}}$ ). The cells were incubated overnight at $37^{\circ} \mathrm{C}$ with $5 \% \mathrm{CO}_{2}$. On the following day, each serum was 2 -fold serially diluted in the culture medium with the first dilution of 1:20. Each serum was tested in duplicates. The diluted serum was incubated with 100-150 fluorescent focus units (FFU) of mNG SARS-CoV-2 at $37^{\circ} \mathrm{C}$ for $1 \mathrm{~h}$ (final dilution range of 1:20 to 1:20,480), after which the serum-virus mixtures were inoculated onto the pre-seeded Vero E6 cell monolayer in 96-well plates. After $1 \mathrm{~h}$ infection, the inoculum was removed and $100 \mu$ of overlay medium (DMEM supplemented with $0.8 \%$ methylcellulose, $2 \% \mathrm{FBS}$, and $1 \% \mathrm{P} / \mathrm{S}$ ) was added to each well. After incubating the plates at $37^{\circ} \mathrm{C}$ for $16 \mathrm{~h}$, raw images of mNG fluorescent foci were acquired using Cytation ${ }^{\mathrm{TM}} 7$ (BioTek) armed with $2.5 \times$ FL Zeiss objective with wide field of view and processed using the software settings (GFP [469,525] threshold 4000, object seletion size $50-1000 \mu \mathrm{m}$ ). The foci in each well were counted and normalized to the non-serum-treated controls to calculate the relative infectivities. The curves of the relative infectivity versus the serum dilutions $\left(\log _{10}\right.$ values) were plotted using Prism 9 (GraphPad). A nonlinear regression method with $\log$ (inhibitor) vs. response-varialbe slope (four parameters) model (bottom and top parameters were constrained to 0 and 100, respectively) was used to determine the dilution fold that neutralized $50 \%$ of mNG SARS-CoV-2 (defined as FFRNT $_{50}$ ) in GraphPad Prism 9.

Statistics. The nonparametric Wilcoxon matched-pairs signed rank test was used to analyze the statistical significance in Fig. 1. 
Reporting summary. Further information on research design is available in the Nature Research Reporting Summary linked to this article.

\section{Data availability}

The raw data that support the findings of this study are shown in the Source data file. Source data are provided with this paper.

Received: 21 December 2021; Accepted: 2 February 2022;

Published online: 09 February 2022

\section{References}

1. Plante, J. A. et al. Spike mutation D614G alters SARS-CoV-2 fitness. Nature, https://doi.org/10.1038/s41586-020-2895-3 (2020).

2. Xie, X. et al. Neutralization of SARS-CoV-2 spike $69 / 70$ deletion, E484K and N501Y variants by BNT162b2 vaccine-elicited sera. Nat. Med. 27, 620-621 (2021).

3. Liu, Y. et al. The N501Y spike substitution enhances SARS-CoV-2 transmission. Nature, In press, https://doi.org/10.1101/2021.03.08.434499 (2021).

4. WHO. Classification of Omicron (B.1.1.529): SARS-CoV-2 Variant of Concern. https://www.who.int/news/item/26-11-2021-classification-ofomicron-(b.1.1.529)-sars-cov-2-variant-of-concern (2021).

5. UK Health Security Agency. SARS-CoV-2 variants of concern and variants under investigation in England. Technical brief 31 (2021).

6. Chen, R. E. et al. Resistance of SARS-CoV-2 variants to neutralization by monoclonal and serum-derived polyclonal antibodies. Nat. Med. 27, 717-726 (2021).

7. $\mathrm{Ku}, \mathrm{Z}$. et al. Molecular determinants and mechanism for antibody cocktail preventing SARS-CoV-2 escape. Nat. Commun., https://doi.org/10.1038/ s41467-41020-20789-41467 (2021).

8. Muruato, A. E. et al. A high-throughput neutralizing antibody assay for COVID-19 diagnosis and vaccine evaluation. Nat. Commun. 11, 4059 (2020).

9. Xie, X. et al. An infectious cDNA clone of SARS-CoV-2. Cell Host Microbe 27, 841-848 e843 (2020)

10. Gudbjartsson, D. F. et al. Humoral immune response to SARS-CoV-2 in Iceland. N. Engl. J. Med. 383, 1724-1734 (2020).

11. Falsey, A. R. et al. SARS-CoV-2 neutralization with BNT162b2 vaccine dose 3 . N. Engl. J. Med., https://doi.org/10.1056/NEJMc2113468 (2021).

12. Chia, W. N. et al. Dynamics of SARS-CoV-2 neutralising antibody responses and duration of immunity: a longitudinal study. Lancet Microbe 2, e240-e249 (2021).

13. Widge, A. T. et al. Durability of responses after SARS-CoV-2 mRNA-1273 vaccination. N. Engl. J. Med. 384, 80-82 (2021).

14. Cele S. et al. SARS-CoV-2 Omicron has extensive but incomplete escape of Pfizer BNT162b2 elicited neutralization and requires ACE2 for infection. MedRxiv, https://doi.org/10.1101/2021.12.08.21267417 (2021)

15. Wilhelm A. et al. Reduced neutralization of SARS-CoV-2 Omicron variant by vaccine sera and monoclonal antibodies. MedRiv, https://doi.org/10.1101/ 2021.12.07.21267432 (2021).

16. Deinirattisai W. et al. Reduced neutralisation of SARS-COV-2 OmicronB.1.1.529 variant by post-immunisation serum. MedRxiv, https://doi.org/ 10.1101/2021.12.10.21267534 (2021)

17. Guardian, T. South Africa: previous infections may explain Omicron hospitalisation rate. https://www.theguardian.com/world/2021/dec/2014/ south-africa-previous-infections-may-explain-omicron-hospitalisation-rate? $\mathrm{fr}=$ operanews (2021).

18. Redd A. D. et al. Minimal cross-over between mutations associated with Omicron variant of SARS-CoV-2 and CD8 $+\mathrm{T}$ cell epitopes identified in COVID-19 convalescent individuals. BioRxiv, https://doi.org/10.1101/ 2021.12.06.471446 (2021).
19. Gruell $\mathrm{H}$. et al. mRNA booster immunization elicits potent neutralizing serum activity against the SARS-CoV-2 Omicron variant. MedRiv, https://doi.org/ 10.1101/2021.12.14.21267769 (2021).

20. Xia, H. et al. Neutralization of Omicron SARS-CoV-2 by 2 or 3 doses of BNT162b2 vaccine. bioRxiv, https://doi.org/10.1101/2022.1101.1121.476344 (2022).

21. Xie, X. et al. Engineering SARS-CoV-2 using a reverse genetic system. Nat. Protoc. 16, 1761-1784 (2021).

\section{Acknowledgements}

We thank colleagues at Pfizer and the University of Texas Medical Branch for helpful discussion. P.-Y.S. was supported by NIH grants HHSN272201600013C, AI134907, AI145617, and UL1TR001439, and awards from the Sealy \& Smith Foundation, the Kleberg Foundation, the John S. Dunn Foundation, the Amon G. Carter Foundation, the Gilson Longenbaugh Foundation, and the Summerfield Robert Foundation. S.C.W. was supported by NIH grant R24 AI120942.

\section{Author contributions}

Conceptualization, X.X., P.R., and P.-Y.S.; Methodology, J.Z., H.X., X.X., C.K., R.R.G.M S.C.W., P.R., and P.-Y.S; Investigation, J.Z., H.X., X.X., C.K., R.R.G.M., S.C.W., P.R., and P.-Y.S; Resources, H.X., S.C.W., P.R., and P.-Y.S; Data Curation, J.Z., H.X., X.X., P.R., and P.-Y.S.; Writing-Original Draft, J.Z., H.X., X.X., R.R., and P.-Y.S; Writing-Review \& Editing, X.X., S.C.W., P.R., and P.-Y.S.; Supervision, X.X., S.C.W., P.R., and P.-Y.S.; Funding Acquisition, S.C.W. and P.-Y.S.

\section{Competing interests}

The authors declare competing interests. X.X. and P.-Y.S. have filed a patent on the reverse genetic system. J.Z., H.X., X.X., and P.-Y.S. received compensation from Pfizer for COVID-19 vaccine development. Other authors declare no competing interests.

\section{Additional information}

Supplementary information The online version contains supplementary material available at https://doi.org/10.1038/s41467-022-28544-w.

Correspondence and requests for materials should be addressed to Ping Ren or Pei-Yong Shi.

Peer review information Nature Communications thanks Robert Carnahan, Stephanie Longet and Nicholas Wohlgemuth for their contribution to the peer review of this work

Reprints and permission information is available at http://www.nature.com/reprints

Publisher's note Springer Nature remains neutral with regard to jurisdictional claims in published maps and institutional affiliations.

Open Access This article is licensed under a Creative Commons Attribution 4.0 International License, which permits use, sharing, adaptation, distribution and reproduction in any medium or format, as long as you give appropriate credit to the original author(s) and the source, provide a link to the Creative Commons license, and indicate if changes were made. The images or other third party material in this article are included in the article's Creative Commons license, unles indicated otherwise in a credit line to the material. If material is not included in the article's Creative Commons license and your intended use is not permitted by statutory regulation or exceeds the permitted use, you will need to obtain permission directly from the copyright holder. To view a copy of this license, visit http://creativecommons.org/ licenses/by/4.0/

(C) The Author(s) 2022 